\title{
Switching from oral atypical antipsychotic monotherapy to paliperidone palmitate once-monthly in non-acute patients with schizophrenia: A prospective, open-label, interventional study
}

\author{
Andreas Schreiner $^{1} \cdot$ Asaf Caspi $^{2} \cdot$ Paul Bergmans $^{3} \cdot$ Pierre Cherubin $^{4} \cdot$ Sofia Keim $^{5}$. \\ Elsa Lara $^{6}$ - Irina Pinchuk ${ }^{7}$ - Daniel Schuepbach ${ }^{8,9} \cdot$ Sajid Suleman $^{10}$. \\ Ludger Hargarter ${ }^{1}$
}

Received: 7 February 2016 / Accepted: 3 September 2016 / Published online: 5 November 2016

(C) The Author(s) 2016. This article is published with open access at Springerlink.com

\begin{abstract}
Rationale Long-acting injectable antipsychotic therapies may offer benefits over oral antipsychotics in patients with schizophrenia.

Objective This study aimed to explore the safety, tolerability, and treatment response of paliperidone palmitate oncemonthly in non-acute but symptomatic adult patients switched from previously unsuccessful monotherapy with frequently used oral atypical antipsychotics.

Methods This was a post hoc analysis of a prospective, interventional, single-arm, international, multicenter, open-label, 6-month study.

Results The patients $(N=472)$ were switched to paliperidone palmitate once-monthly (PP1M) from daily oral treatment with either aripiprazole $(n=46)$, olanzapine $(n=87)$, paliperidone extended-release $(n=104)$, quetiapine $(n=44)$, or risperidone $(n=191)$. In all groups, mean Positive and Negative Syndrome Scale total $(p<0.0001)$ and Clinical
\end{abstract}

Electronic supplementary material The online version of this article (doi:10.1007/s00213-016-4445-0) contains supplementary material, which is available to authorized users.

Andreas Schreiner

aschrein@its.jnj.com

1 Medical \& Scientific Affairs, Janssen Cilag EMEA, Johnson \& Johnson Platz 1, 41470 Neuss, Germany

2 Psychiatric Ambulatory Clinic, Sheba Medical Center, Ramat Gan, Israel

3 Biometrics and Reporting, Janssen Cilag Benelux, Tilburg, The Netherlands

4 Medical Affairs, Janssen Cilag EMEA, Issy-les-Moulineaux, France
Global Impression-Severity scores improved significantly ( $p=0.0004$ to $p<0.0001$ ). An improvement of $\geq 50 \%$ in the Positive and Negative Syndrome Scale total score was observed in $21.7 \%$ (aripiprazole), $29.9 \%$ (olanzapine), $29.8 \%$ (paliperidone extended-release), $27.3 \%$ (quetiapine), and $37.2 \%$ (risperidone) of patients. The patients showed significant improvements in the Personal and Social Performance score (aripiprazole $p=0.0409$, all others $p \leq 0.0015$ ); Mini International Classification of Functionality, Disability and Health Rating for Activity and Participation Disorders in Psychological Illnesses total scores (all $p<0.01$ ); and Treatment Satisfaction Questionnaire for Medication Global Satisfaction score (olanzapine and risperidone $p<0.0001$, quetiapine $p=0.0465$, paliperidone extended-release $p=0.0571$, aripiprazole $p=\mathrm{NS}$ ). Paliperidone palmitate oncemonthly was well tolerated, presenting no new safety signals. Conclusions These data illustrate that stable, non-acute but symptomatic patients on oral antipsychotic monotherapy

Global Clinical Operations EMEA MAO, Janssen Cilag, Barcarena, Portugal

6 Hospital CUF Infante Santo, Lisbon, Portugal

7 Ukrainian Research Institute of Social and Forensic Psychiatry and Drug Abuse, Ministry of Health of Ukraine, Kiev, Ukraine

8 Department of Psychiatry, Psychotherapy and Psychosomatics, University Hospital of Psychiatry Zurich, Zurich, Switzerland

9 Klinikum am Weissenhof, Weinsberg, Germany

10 South London \& Maudsley NHS Foundation Trust, Ladywell Unit, University Hospital Lewisham, London, UK 
may show clinically meaningful improvement of symptoms, functioning, and treatment satisfaction after direct transition to PP1M. The findings are limited by the naturalistic study design; thus, further studies are required to confirm the current findings.

Keywords Functioning $\cdot$ Non-acute $\cdot$ Long-acting injectable antipsychotic therapy $\cdot$ Oral antipsychotic $\cdot$ Paliperidone palmitate $\cdot$ Switching $\cdot$ Schizophrenia $\cdot$ Treatment satisfaction

\section{Introduction}

Pharmacotherapy, which includes oral and long-acting injectable (LAI) antipsychotics, remains the mainstay treatment in schizophrenia (Hasan et al. 2013); nevertheless, discontinuation rates are high (Kahn et al. 2008; Lieberman et al. 2005; Naber and Lambert 2009). Clinical practice guidelines strongly recommended antipsychotic monotherapy for the treatment of schizophrenia (Barnes 2011; Lerma-Carrillo et al. 2008; National Institute for Health and Care Excellence (NICE) 2014).

Oral atypical antipsychotics generally have the same overall efficacy but differ in their side effect profiles, with apparent differences in sedation, metabolic disturbances such as weight gain, glucose and lipid abnormalities, and the risk of extrapyramidal motor symptoms (EPMSs) (Davis et al. 2003; De Hert et al. 2012; Jones et al. 2010; Leucht et al. 2013; RummelKluge et al. 2010). Non-adherence to antipsychotic medication is associated with increased risk of relapse and hospitalization at all stages of schizophrenia (Kozma and Weiden 2009; Leucht and Heres 2006; Llorca 2008; Robinson et al. 1999), and may be influenced by several factors (Tandon et al. 2006), including poor efficacy (worsening of symptoms) (Liu-Seifert et al. 2005) and the presence of side effects (Higashi et al. 2013).

LAI antipsychotic therapy (LAT) may improve adherence to medication in patients with schizophrenia (Cañas et al. 2013) and, as a consequence, significantly reduce relapse rates and improve long-term outcomes compared with those treated with oral antipsychotic medication (Leucht et al. 2011; Tiihonen et al. 2011). A systematic review and meta-analysis of mirror-image studies comparing a period of treatment using oral antipsychotics with a subsequent period of treatment using LAT within the same patient showed that LATs were significantly superior in reducing relapse rates compared with oral antipsychotics (Kishimoto et al. 2013); however, this outcome was in contrast to a recent meta-analysis based on randomized controlled trials (RCTs) comparing LATs and oral antipsychotics (Kishimoto et al. 2014). The short duration of the majority of RCTs, the very defined patient inclusion criteria, and the added care and close follow-up of patients suggest that such studies do not adequately reflect real clinical practice or treatment of patients with schizophrenia in the community, and therefore longer, naturalistic studies are required (Olivares et al. 2009b). Consequently, it has been shown that the outcomes of studies comparing oral antipsychotics and LATs are sensitive to trial design, and RCTs are not optimal for exploring differences between oral antipsychotics and LATs (Alphs et al. 2014; Kirson et al. 2013).

Since treatment response and side effect profiles vary between different antipsychotics (Jones et al. 2010; Leucht et al. 2013; Taylor et al. 2012), it is of particular interest to explore treatment response, safety, and tolerability in patients previously unsuccessfully treated with oral atypical antipsychotic monotherapy who were switched to LAT in a routine clinical setting. Paliperidone palmitate once-monthly (PP1M) is an atypical LAT designed for intramuscular (IM) administration indicated for the maintenance treatment of adult patients with schizophrenia (Janssen Cilag 2015).

The Paliperidone Palmitate Flexible Dosing in Schizophrenia (PALMFlexS) study was a prospective, 6month, pragmatic, interventional study conducted in a large, more representative sample of patients with schizophrenia than those recruited in the pivotal RCTs (Hargarter et al. 2015; Schreiner et al. 2015b; Schreiner et al. 2014a). The study was designed specifically to reflect more closely realworld clinical situations in which the transition to another antipsychotic is performed in previously unsuccessfully treated patients. The PALMFlexS study included three distinct patient populations: patients with non-acute schizophrenia switching to PP1M from oral antipsychotics, non-acute patients switching to PP1M from other LATs, and acute patients switching to PP1M from oral antipsychotics.

To understand the impact of dosing and switching strategies when initiating PP1M, the current analysis was conducted in non-acute but symptomatic patients with schizophrenia switched from previously unsuccessful monotherapy with the most frequently used oral atypical antipsychotics.

\section{Materials and methods}

This was a post hoc analysis of a prospective, interventional, single-arm, multicenter, open-label, 6-month study performed in patients with schizophrenia from 160 centers across 21 countries between November 2010 and November 2012 (NCT01281527). The study was performed in accordance with the Declaration of Helsinki and was consistent with Good Clinical Practices of the International Conference on Harmonisation and applicable regulatory requirements. All the patients provided informed written consent.

The protocol, full details of the study population, and overall results have previously been reported (Schreiner et al. 
2014a). Methods that are specific to this post hoc analysis are briefly described below.

\section{Study design and patients}

Non-acute but symptomatic male or female patients aged $\geq 18$ years with schizophrenia (diagnosed according to the Diagnostic and Statistical Manual of Mental Disorders, Fourth Edition criteria), who were previously unsuccessfully treated using oral monotherapy with either aripiprazole (ARI), olanzapine (OLA), paliperidone extended-release (Pali ER), quetiapine (QUE), or risperidone (RIS), were selected from the overall study population of PALMFlexS (Schreiner et al. 2014a).

The patients were required to be "stable" but symptomatic, i.e., have been on the same oral atypical antipsychotic monotherapy for the treatment of schizophrenia on an adequate therapeutic dose and with a change in the Clinical Global Impression-Severity (CGI-S) score of $\leq 1$ for $\geq 4$ weeks prior to enrolment. Their current treatment was considered to have been unsuccessful due to one or more of the following reasons: lack of efficacy (baseline Positive and Negative Syndrome Scale [PANSS] total score $\geq 70$ or $\geq 2$ items scoring $\geq 4$ in the PANSS positive or negative subscale or $\geq 3$ items scoring $\geq 4$ in the PANSS general psychopathology subscale, as judged by the investigator), lack of tolerability or safety (defined as the presence of intolerable [according to the patient] and/or clinically relevant [according to the investigator] side effects on their current antipsychotic medication), lack of adherence, or patient's wish. Lack of adherence was assessed individually by the investigator. There were no specific protocol-defined criteria. Patients were excluded if, at the discretion of the investigator, their diagnosis was considered to be the direct result of the pharmacological effects of a substance or general medical condition, they were treatment naïve, they had received clozapine within 3 months prior to the start of the study, they were considered at imminent risk of suicide even after clinical intervention, they had a history of or current symptoms of tardive dyskinesia or neuroleptic malignant syndrome, they were pregnant or breastfeeding, or they had any known allergies to RIS or paliperidone or any of its excipients. The inclusion/exclusion criteria were designed to recruit a more diverse study population than those in previously conducted pivotal studies (Gopal et al. 2010; Pandina et al. 2010); for example, patients with relevant comorbidities, co-medications, and current substance use or abuse, with the exception of intravenous drug use, were eligible for enrolment and there were no exclusions based on body mass index (BMI).

In the present study, the patients were switched directly to PP1M, in line with the indication and posology of PP1M European summary of product characteristics (Janssen Cilag 2015). After initiation of PP1M, the patients were tapered off their oral atypical antipsychotic at the discretion of the treating physician, preferably within a maximum of 4 weeks. PP1M was initiated, in line with the $\mathrm{SmPC}$, at a recommended dose of $150 \mathrm{mg}$ equivalent (mg eq) on day 1 and $100 \mathrm{mg}$ eq on day 8 ( \pm 2 days; nota bene, the current summary of product characteristics now states \pm 4 days) intramuscularly, both given in the deltoid muscle. Subsequently, PP1M was administered once-monthly ( \pm 7 days) (visit days) using flexible maintenance dosages within the range of 50 to $150 \mathrm{mg}$ eq based on the clinical judgment of the treating physician. Patients without documentation of previous RIS or paliperidone exposure were tested for tolerability with Pali ER (3 $\mathrm{mg} /$ day) for at least 2 days prior to receiving PP1M. Efficacy, tolerability, and safety were assessed by the same person at each study visit, whenever possible.

\section{Efficacy assessments}

Efficacy outcomes were assessed after 6 months of treatment by trained, qualified, non-blinded assessors. Evaluation time points were days 1 (baseline), 8, 38 (month 1), 68 (month 2), 98 (month 3), 128 (month 4), 158 (month 5), and 188 (month 6 ). The primary efficacy outcome for non-acute but symptomatic patients with schizophrenia switched due to lack of efficacy was the percentage of patients achieving treatment response, defined as $\geq 20 \%$ improvement in PANSS total score from baseline to endpoint ( 6 months or time of early discontinuation). Maintained efficacy (defined as non-inferiority in the change in PANSS total score at endpoint versus baseline, as measured by means of Schuirmann's test) was the primary efficacy outcome for patients switched to PP1M for other reasons. Actual scores and change from baseline in CGI-S score, Personal and Social Performance (PSP) scale (Morosini et al. 2000) total score, Mini International Classification of Functionality, Disability and Health (ICF) Rating for Activity and Participation Disorders in Psychological Illnesses (Mini-ICF-APP) (Linden and Baron 2005; Molodynski et al. 2013), and treatment satisfaction (assessed in patients using the 14-item Treatment Satisfaction Questionnaire for Medication [TSQM] scale (Atkinson et al. 2004) and physician treatment satisfaction using a 7-point categorical scale) scores were also analyzed.

\section{Safety and tolerability}

All treatment-emergent adverse events (TEAEs), defined as adverse events that were new in onset or were aggravated in severity following initiation of PP1M, were documented at each clinic visit and coded using the Medical Dictionary for Regulatory Activities (version 13.0). EPMSs were assessed by the Extrapyramidal Symptom Rating Scale (ESRS) (Chouinard and Margolese 2005). In addition, alcohol and substance use were measured using the Clinician Rating Alcohol Use Scale (CRAUS) and the Clinician Rating Substance Use Scale (CRSUS) (Carey et al. 1996). Body 
weight was recorded at each assessment point and endpoint, and BMI was calculated. There were no obligatory protocolbased prolactin measurements; however, investigators were allowed to measure prolactin levels at any time during the study at their own discretion.

\section{Data analysis}

The intent-to-treat (ITT) population comprised all patients who received PP1M at least once. Analysis of treatment response was performed on the efficacy analysis population, which included all ITT patients with at least one post-baseline efficacy assessment. Endpoint analysis using the last observation carried forward (LOCF) method was performed in addition to observed case analysis. Actual values and changes from baseline were summarized descriptively at each assessment time point and at the patient's last evaluation (endpoint) while categorical variables were summarized with frequency and percentage. Within-group changes in efficacy parameters from baseline to endpoint were analyzed using the Wilcoxon signed-rank test. Between-oral-subgroup differences were tested using Fisher's exact test and the Kruskal-Wallis test. All tests were performed using Statistical Analysis System version 9.2.

Safety and tolerability were evaluated throughout the study on the safety ITT population, which comprised all ITT patients who had at least one post-baseline safety observation. TEAE frequency distributions included severity of events (i.e., mild, moderate, or severe) and causal relationship to treatment (i.e., not related, doubtful, possible, probably, or very likely).

\section{Results}

\section{Demographics and patient disposition}

In total, 472 non-acute patients with schizophrenia were eligible for this analysis. Patients enrolled in the study were on a stable oral atypical antipsychotic dose at enrolment (Table 1). The reasons for patients to switch from their current oral atypical antipsychotic to PP1M were patient's wish (45\%), lack of efficacy $(22 \%)$, and lack of compliance $(25 \%)$.

Patient disposition is described in Fig. 1. Baseline characteristics are summarized in Table 1. There were nonsignificant between-group differences in mean age (standard deviation [SD]; ranging from 34.4 [9.4] years [ARI] to 40.8 [11.7] years [QUE]), mean body weight (79.7 [17.3] kg [RIS] to 89.1 [22.1] $\mathrm{kg}$ [ARI]), and percentage of males in the group (50.0\% [QUE] to $73.9 \%$ [ARI]) at baseline (Table 1). At baseline, $39(9.3 \%)$ patients were reported to have a diagnosis of substance abuse (with or without impairment).

Following the day $1(150 \mathrm{mg}$ eq $) /$ day $8(100 \mathrm{mg} \mathrm{eq})$ initiation regimen, the PP1M mean modal maintenance dose from the third injection onwards ranged from 94.9 (35.0) $\mathrm{mg}$ eq for patients who switched from ARI to 105.0 (36.8) $\mathrm{mg}$ eq for patients who switched from QUE; the final dose distribution of PP1M is summarized in Table 1. Regardless of the oral antipsychotic that patients were switched from, most $(92.3 \%$ [Pali ER] to $97.7 \%$ [QUE]) received PP1M according to the recommended initiation regimen. Overall, $72.8 \%$ of patients had a dose adjustment after the third dose of PP1M, with the majority requiring only one adjustment (ARI $45.7 \%$, OLA $54.0 \%$, Pali ER $49.0 \%$, QUE $45.5 \%$, RIS $50.8 \%$ ). The percentage of patients with one or more dose increase varied from $36.5 \%$ (Pali ER) to $45.5 \%$ (QUE), and the percentage of patients with one or more dose decrease varied from $38.6 \%$ (QUE) to $55.8 \%$ (Pali ER).

The proportion of patients using concomitant medications (benzodiazepines and anticholinergics) during the study is shown in Table 1.

\section{Efficacy outcomes}

At endpoint, of all patients that switched from previous atypical oral monotherapy to PP1M, 52.2 \% (ARI), $60.9 \%$ (OLA), $57.7 \%$ (Pali ER), $65.9 \%$ (QUE), and $73.8 \%$ (RIS) had a $\geq 20 \%$ improvement in PANSS total score. In addition, $21.7 \%$ (ARI), $29.9 \%$ (OLA), $29.8 \%$ (Pali ER), $27.3 \%$ (QUE), and $37.2 \%$ (RIS) of patients had a $\geq 50 \%$ improvement in PANSS total score. The mean PANSS total score was significantly improved from baseline to endpoint in all groups $(p<0.0001)$ (Fig. 2). Disease severity, as measured by the mean CGI-S score, improved significantly from baseline to endpoint in all groups $(p=0.0004$ to $p<0.0001)$ (Table 2).

\section{Secondary outcomes}

At endpoint, patients who were switched from atypical oral antipsychotics to PP1M showed a statistically significant improvement from baseline in PSP total scores $(p=0.0409$ for ARI to $p<0.0001$ for RIS, Pali ER, and QUE) and in Mini-ICFAPP total score $(p=0.0079$ for ARI to $p<0.0001$ for RIS and Pali ER) (Table 2). Significant improvements from baseline to endpoint in TSQM Global Satisfaction score were observed in patients who switched from OLA $(p<0.0001)$, QUE $(p=0.0465)$, and RIS $(p<0.0001)$ and in patients who switched from Pali ER ( $p=0.0571)$. There was no significant difference in TSQM Global Satisfaction score between ARI and PP1M. Overall, physician treatment satisfaction improved significantly from baseline to endpoint in all groups $(p<0.0001)$ (Table 2).

\section{Safety and tolerability}

TEAEs affecting $\geq 5 \%$ of patients in any group are summarized in Table 3. Mean change in the ESRS total score (SD) from baseline to endpoint was -0.6 (3.4) for ARI, -1.3 (4.4) for OLA, -0.7 (4.1) for Pali ER, -0.3 (3.2) for QUE, and -1.2 (3.5) for RIS $(p<0.05$ for all, except QUE $p=0.4857)$ 
Table 1 Baseline characteristics and PP1M dosing $(N=472)$

\begin{tabular}{|c|c|c|c|c|c|c|}
\hline Patients switched to PP1M from & ARI & OLA & Pali ER & QUE & RIS & $p$ value \\
\hline ITT population, $n$ & 46 & 87 & 104 & 44 & 191 & \\
\hline Mean age, years (SD) & $34.4(9.4)$ & $36.8(11.6)$ & $37.7(11.7)$ & $40.8(11.7)$ & $38.7(12.5)$ & $0.0773^{\mathrm{a}}$ \\
\hline Male, $\%$ & 73.9 & 67.8 & 70.2 & 50.0 & 61.8 & $0.0861^{\mathrm{b}}$ \\
\hline Mean age at diagnosis, years (SD) & $27.6(6.7)$ & $28.4(9.7)$ & $29.0(9.5)$ & $30.7(10.3)$ & $30.7(10.4)$ & \\
\hline Diagnosis of paranoid schizophrenia, $\%$ & 78.3 & 79.3 & 81.7 & 72.7 & 74.9 & \\
\hline Mean baseline weight, $\mathrm{kg}(\mathrm{SD})$ & $89.1(22.1)$ & $79.8(16.4)$ & $80.0(17.3)$ & $80.4(16.0)$ & $79.7(17.3)$ & $0.1018^{\mathrm{a}}$ \\
\hline Mean baseline BMI, kg/m² (SD; range) & $29.9(7.5 ; 17,51)$ & $27.2(6.1 ; 18,46)$ & $27.0(5.5 ; 17,51)$ & $28.1(5.3 ; 18,39)$ & $27.0(5.5 ; 17,54)$ & $0.1120^{\mathrm{a}}$ \\
\hline Patients with $\geq 1$ comorbidity, $\%^{\mathrm{c}}$ & 78.3 & 63.2 & 58.7 & 52.3 & 59.2 & \\
\hline \multicolumn{7}{|l|}{ Number of previous hospitalizations, $\%$} \\
\hline None & 10.9 & 18.4 & 21.2 & 18.2 & 19.9 & \\
\hline $1-3$ & 45.7 & 47.1 & 35.6 & 36.4 & 48.7 & \\
\hline$\geq 4$ & 43.5 & 34.5 & 43.3 & 45.5 & 31.4 & \\
\hline $\begin{array}{l}\text { Patients with diagnosed substance abuse } \\
\text { (with or without impairment), \% }\end{array}$ & 9.8 & 10.5 & 6.4 & 5.4 & 11.1 & \\
\hline $\begin{array}{l}\text { Mean daily dose of prior antipsychotic, } \\
\text { mg (SD) }\end{array}$ & $22.7(10.7)$ & $15.6(8.2)$ & $7.6(2.6)$ & $482.4(277.1)$ & $4.3(2.3)$ & \\
\hline $\begin{array}{l}\text { Patients receiving PP1M initiation regimen } \\
\text { at day } 1 \text { and day } 8 \text { according to label, } \% \text { d }\end{array}$ & 93.5 & 95.4 & 92.3 & 97.7 & 96.3 & \\
\hline $\begin{array}{l}\text { Mean modal PP1M maintenance dose, } \\
\text { mg eq }(\mathrm{SD})^{\mathrm{e}}\end{array}$ & $94.9(35.0)$ & $104.2(33.6)$ & $100.5(32.3)$ & $105.0(36.8)$ & $98.9(32.3)$ & \\
\hline \multicolumn{7}{|l|}{ Last PP1M dose received, $\%$ of patients } \\
\hline $50 \mathrm{mg} \mathrm{eq}$ & 19.6 & 6.9 & 8.7 & 13.6 & 8.4 & \\
\hline $75 \mathrm{mg}$ eq & 19.6 & 32.2 & 32.7 & 15.9 & 35.1 & \\
\hline $100 \mathrm{mg} \mathrm{eq}$ & 30.4 & 31.0 & 35.6 & 40.9 & 33.0 & \\
\hline $150 \mathrm{mg} \mathrm{eq}$ & 30.4 & 29.9 & 23.1 & 29.5 & 23.6 & \\
\hline \multicolumn{7}{|l|}{ Relevant co-medications } \\
\hline \multicolumn{7}{|l|}{ Number (\%) of patients using benzodiazepines } \\
\hline At baseline & $12(26.1)$ & $25(28.7)$ & $28(26.9)$ & $9(20.5)$ & $39(20.4)$ & \\
\hline Newly initiated during study & $11(23.9)$ & $21(24.1)$ & $26(25.0)$ & $14(31.8)$ & $30(15.7)$ & \\
\hline At endpoint & $12(26.1)$ & $21(24.1)$ & $21(20.2)$ & $8(18.2)$ & $36(18.8)$ & \\
\hline At 6 months for completers ${ }^{\mathrm{f}}$ & $9(29.0)$ & $10(17.5)$ & $15(18.1)$ & $6(18.8)$ & $29(18.2)$ & \\
\hline \multicolumn{7}{|l|}{ Number $(\%)$ of patients using anticholinergics } \\
\hline At baseline & $2(4.3)$ & $8(9.2)$ & $10(9.6)$ & $4(9.1)$ & $23(12.0)$ & \\
\hline Newly initiated during study & $2(4.3)$ & $9(10.3)$ & $7(6.7)$ & $4(9.1)$ & $14(7.3)$ & \\
\hline At endpoint & $2(4.3)$ & $8(9.2)$ & $5(4.8)$ & $4(9.1)$ & $14(7.3)$ & \\
\hline At 6 months for completers ${ }^{\mathrm{f}}$ & $2(6.5)$ & $5(8.8)$ & $4(4.8)$ & $2(6.3)$ & $12(7.5)$ & \\
\hline
\end{tabular}

$A R I$ aripiprazole, $B M I$ body mass index, ITT intent to treat, $O L A$ olanzapine, Pali ER paliperidone extended-release, $P P 1 M$ once-monthly paliperidone palmitate, $Q U E$ quetiapine, $R I S$ risperidone, $S D$ standard deviation. $p$ values indicate differences between prior oral antipsychotic treatment subgroups

${ }^{a}$ Kruskal-Wallis test

${ }^{\mathrm{b}}$ Fisher's exact test

${ }^{\mathrm{c}}$ Individual patients can be labelled for $>1$ comorbidity

${ }^{\mathrm{d}}$ The recommended initiation regimen was PP1M $150 \mathrm{mg}$ eq on day 1 and $100 \mathrm{mg}$ eq on day 8 , given in the deltoid muscle

${ }^{\mathrm{e}}$ Excluding the initiation regimen (day $1 /$ day 8 )

${ }^{\mathrm{f}} \mathrm{ARI} n=31$; OLA $n=57$; Pali ER $n=83$; QUE $n=32$; RIS $n=159$

(Fig. 3). Mean weight change (SD) from baseline to endpoint ranged between $-0.3(4.6) \mathrm{kg}$ for OLA (95\% confidence interval $[\mathrm{CI}]-1.3,0.7)$ and $3.5(6.3) \mathrm{kg}$ for ARI $(95 \% \mathrm{CI}$ $1.5,5.4$ ) (Supplementary Table 1). Mean changes in BMI from baseline to endpoint were similar, ranging from $-0.1(1.6) \mathrm{kg} / \mathrm{m}^{2}$ for OLA $(95 \% \mathrm{CI}-0.5,0.3)$ to 1.2 (2.2) $\mathrm{kg} / \mathrm{m}^{2}$ for ARI $(95 \%$ CI $0.5,1.9)$ (Supplementary Table 1). 


\begin{tabular}{|c|c|c|c|c|c|}
\hline & \multicolumn{5}{|c|}{$\begin{array}{l}\text { All patients switching from previous } \\
\text { unsuccessful monotherapy }(\mathrm{N}=472)\end{array}$} \\
\hline & $\downarrow$ & $\downarrow$ & $\downarrow$ & $\downarrow$ & $\downarrow$ \\
\hline Patients switched to PP1M from: & ARI & OLA & Pali ER & QUE & RIS \\
\hline Patients enrolled, $n$ & 46 & 87 & 104 & 44 & 191 \\
\hline ITT population*, $\mathrm{n}$ & 46 & 87 & 104 & 44 & 191 \\
\hline & $\downarrow$ & $\downarrow$ & $\downarrow$ & $\downarrow$ & $\downarrow$ \\
\hline Patients switched to PP1M from: & $\overline{A R I}$ & $\overline{\text { OLA }}$ & $\overline{\text { Pali ER }}$ & $\overline{\text { QUE }}$ & RIS \\
\hline Study completers, $\%$ & 67.4 & 65.5 & 79.8 & 72.7 & 83.2 \\
\hline \multicolumn{6}{|l|}{ Reasons for discontinuation, $\%$} \\
\hline Withdrew consent & 13.0 & 11.5 & 6.7 & 11.4 & 5.2 \\
\hline Adverse event & 15.2 & 5.7 & 2.9 & 9.1 & 3.1 \\
\hline Lost to follow-up & 2.2 & 5.7 & 2.9 & 4.5 & 1.6 \\
\hline Lack of efficacy & 0.0 & 4.6 & 3.8 & 0.0 & 2.1 \\
\hline Physician decision & 2.2 & 0.0 & 1.9 & 0.0 & 2.1 \\
\hline Non-compliance with study drug & 0.0 & 1.1 & 1.0 & 0.0 & 1.0 \\
\hline Protocol violation & 0.0 & 3.4 & 0.0 & 0.0 & 0.5 \\
\hline Other & 0.0 & 2.3 & 1.0 & 2.3 & 0.5 \\
\hline
\end{tabular}

Fig. 1 Patient disposition. *patients who received at least one dose of study drug. ARI aripiprazole, ITT intent to treat, OLA olanzapine, PPIM oncemonthly paliperidone palmitate, Pali ER paliperidone extended-release, $Q U E$ quetiapine, $R I S$ risperidone

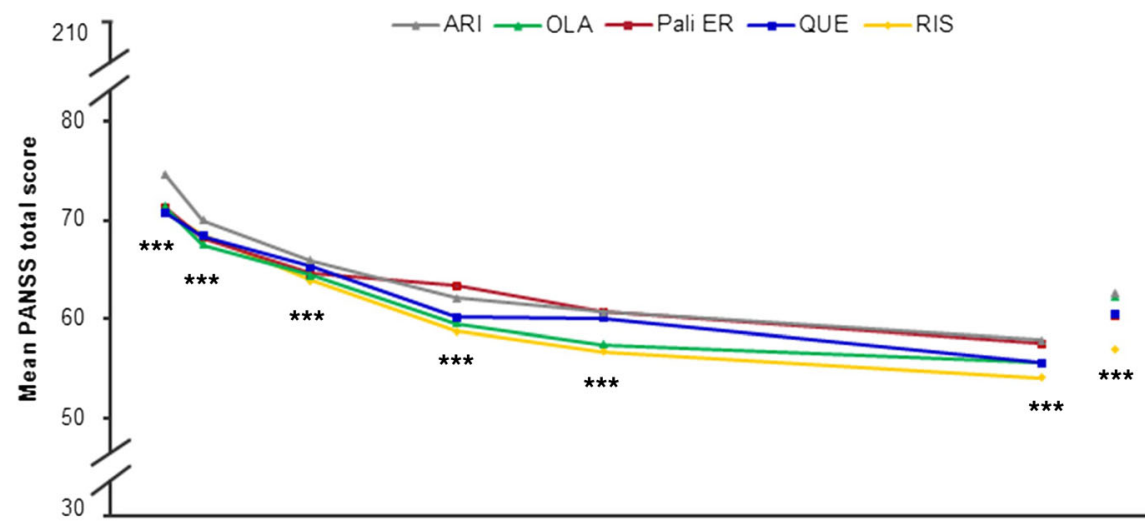

\begin{tabular}{|l|c|c|c|c|c|c|c|c|}
\hline $\begin{array}{l}\text { Patients switched } \\
\text { to PP1M from: }\end{array}$ & $\begin{array}{c}\text { Day 1 } \\
\mathbf{( B L )}\end{array}$ & $\begin{array}{c}\text { Day } \\
\mathbf{8}\end{array}$ & $\begin{array}{c}\text { Month } \\
\mathbf{1}\end{array}$ & $\begin{array}{c}\text { Month } \\
\mathbf{2}\end{array}$ & $\begin{array}{c}\text { Month } \\
\mathbf{3}\end{array}$ & $\mathbf{3}$ & $\begin{array}{c}\text { Month } \\
\mathbf{6}\end{array}$ & Endpoint \\
\hline ARI, $\mathrm{n}$ & 46 & 46 & 43 & 39 & 38 & & 31 & 46 \\
\hline OLA, $\mathrm{n}$ & 87 & 87 & 82 & 76 & 71 & & 54 & 87 \\
\hline Pali ER, $\mathrm{n}$ & 104 & 103 & 101 & 97 & 93 & & 83 & 104 \\
\hline QUE, $\mathrm{n}$ & 44 & 44 & 43 & 37 & 37 & & 32 & 44 \\
\hline RIS, $\mathrm{n}$ & 191 & 190 & 187 & 179 & 168 & & 159 & 191 \\
\hline
\end{tabular}

Fig. 2 Mean PANSS total score over time (efficacy ITT population; $N=472$ ). $p<0.001$ vs baseline for all antipsychotics. $A R I$ aripiprazole, $B L$ baseline, ITT intent to treat, OLA olanzapine, PANSS Positive and
Negative Syndrome Scale, PPIM once-monthly paliperidone palmitate, Pali ER paliperidone extended-release, $Q U E$ quetiapine, RIS risperidone. $* * * p<0.0001$ vs baseline for all antipsychotics 
Table 2 Secondary efficacy outcomes (efficacy ITT population; $N=472$ )

\begin{tabular}{|c|c|c|c|c|c|}
\hline Patients switched to PP1M from & ARI & OLA & Pali ER & QUE & RIS \\
\hline Mean PANSS total score, $n$ & 44 & 87 & 104 & 44 & 191 \\
\hline Baseline (SD) & 74.7 (14.9) & $71.4(13.2)$ & $71.3(14.3)$ & $70.8(13.1)$ & $70.8(15.1)$ \\
\hline Endpoint (SD) & $62.6(16.5)$ & $62.3(19.6)$ & $60.4(17.2)$ & $60.5(20.1)$ & $56.9(17.3)$ \\
\hline $\begin{array}{l}\text { Mean change from baseline } \\
\text { to endpoint }(\mathrm{SD})\end{array}$ & $-12.2(16.7)$ & $-9.1(17.5)$ & $-10.8(14.4)$ & $-10.2(19.6)$ & $-13.9(14.8)$ \\
\hline $\begin{array}{l}95 \% \text { CI of mean change } \\
\left(p \text { value }^{\mathrm{b}}\right)\end{array}$ & $\begin{array}{l}-17.1,-7.2 \\
(<0.0001)\end{array}$ & $\begin{array}{l}-12.9,-5.4 \\
(<0.0001)\end{array}$ & $\begin{array}{l}-13.6,-8.0 \\
(<0.0001)\end{array}$ & $\begin{array}{r}-16.2,-4.3 \\
(<0.0001)\end{array}$ & $\begin{array}{l}-16.1,-11.8 \\
(<0.0001)\end{array}$ \\
\hline Mean CGI-S score, $n^{\text {a }}$ & 46 & 86 & 104 & 44 & 189 \\
\hline Baseline (SD) & $4.1(0.8)$ & $3.7(1.0)$ & $3.9(0.9)$ & $3.9(0.9)$ & $3.8(0.9)$ \\
\hline Endpoint (SD) & $3.5(1.0)$ & $3.3(1.2)$ & $3.4(1.1)$ & $3.4(1.0)$ & $3.0(1.0)$ \\
\hline $\begin{array}{l}\text { Mean change from baseline } \\
\text { to endpoint (SD) }\end{array}$ & $-0.6(1.1)$ & $-0.4(1.1)$ & $-0.6(1.1)$ & $-0.5(1.1)$ & $-0.8(0.9)$ \\
\hline $\begin{array}{l}95 \% \text { CI of mean change } \\
\left(p \text { value }^{\mathrm{b}}\right)\end{array}$ & $\begin{array}{l}-0.9,-0.3 \\
(0.0003)\end{array}$ & $\begin{array}{l}-0.6,-0.2 \\
(0.0003)\end{array}$ & $\begin{array}{l}-0.8,-0.4 \\
(<0.0001)\end{array}$ & $\begin{array}{l}-0.9,-0.2 \\
(0.0004)\end{array}$ & $\begin{array}{l}-0.9,-0.7 \\
(<0.0001)\end{array}$ \\
\hline Mean PSP score, $n^{\text {a }}$ & 44 & 84 & 103 & 44 & 185 \\
\hline Baseline (SD) & $58.9(13.4)$ & $61.5(14.6)$ & $58.3(13.7)$ & $56.3(12.0)$ & $57.8(12.3)$ \\
\hline Endpoint (SD) & $62.9(15.2)$ & $66.0(17.7)$ & $65.4(16.4)$ & $64.2(15.9)$ & $68.2(13.9)$ \\
\hline $\begin{array}{l}\text { Mean change from baseline } \\
\text { to endpoint (SD) }\end{array}$ & $3.9(13.2)$ & $4.5(15.9)$ & $7.0(13.8)$ & $7.9(12.4)$ & $10.4(13.8)$ \\
\hline $\begin{array}{l}95 \% \text { CI of mean change } \\
\left(p \text { value }^{\mathrm{b}}\right)\end{array}$ & $\begin{array}{r}-0.1,8.0 \\
(0.0409)\end{array}$ & $\begin{array}{l}1.1,8.0 \\
(0.0015)\end{array}$ & $\begin{array}{l}4.3,9.7 \\
(<0.0001)\end{array}$ & $\begin{array}{l}4.1,11.6 \\
(<0.0001)\end{array}$ & $\begin{array}{l}8.4,12.4 \\
(<0.0001)\end{array}$ \\
\hline Mean Mini-ICF-APP total score, $n^{\mathrm{c}}$ & 43 & 79 & 97 & 42 & 179 \\
\hline Baseline (SD) & $19.0(7.8)$ & $18.1(8.8)$ & $19.9(8.5)$ & $21.6(6.9)$ & $19.9(7.0)$ \\
\hline Endpoint (SD) & $16.1(9.8)$ & $15.3(9.5)$ & $16.8(9.6)$ & $17.8(8.7)$ & $14.7(7.3)$ \\
\hline $\begin{array}{l}\text { Mean change from baseline } \\
\text { to endpoint (SD) }\end{array}$ & $-2.9(7.1)$ & $-2.8(7.8)$ & $-3.1(7.3)$ & $-3.8(9.2)$ & $-5.2(7.1)$ \\
\hline $\begin{array}{l}95 \% \text { CI of mean change } \\
\left(p \text { value }^{\mathrm{b}}\right)\end{array}$ & $\begin{array}{c}-5.1,-0.7 \\
(0.0079)\end{array}$ & $\begin{array}{l}-4.5,-1.1 \\
(0.0013)\end{array}$ & $\begin{array}{l}-4.5,-1.6 \\
(<0.0001)\end{array}$ & $\begin{array}{l}-6.7,-0.9 \\
(0.0015)\end{array}$ & $\begin{array}{l}-6.3,-4.2 \\
(<0.0001)\end{array}$ \\
\hline Physician overall treatment satisfaction, $n$ & 41 & 76 & 94 & 38 & 172 \\
\hline Baseline (SD) & $4.5(1.1)$ & $4.1(1.2)$ & $3.1(1.0)$ & $4.1(1.1)$ & $3.9(1.1)$ \\
\hline Endpoint (SD) & $2.8(1.1)$ & $2.7(1.2)$ & $2.4(1.0)$ & $2.7(1.3)$ & $2.2(0.9)$ \\
\hline $\begin{array}{l}\text { Mean change from baseline } \\
\text { to endpoint (SD) }\end{array}$ & $-1.7(1.5)$ & $-1.4(1.6)$ & $-0.7(1.3)$ & $-1.4(1.6)$ & $-1.7(1.4)$ \\
\hline $\begin{array}{l}95 \% \text { CI of mean change } \\
\left(p \text { value }^{\mathrm{b}}\right)\end{array}$ & $\begin{array}{l}-2.1,-1.2 \\
(<0.0001)\end{array}$ & $\begin{array}{l}-1.7,-1.0 \\
(<0.0001)\end{array}$ & $\begin{array}{r}-0.9,-0.4 \\
(<0.0001)\end{array}$ & $\begin{array}{r}-1.9,-0.9 \\
(<0.0001)\end{array}$ & $\begin{array}{l}-1.9,-1.5 \\
(<0.0001)\end{array}$ \\
\hline $\begin{array}{l}\text { Patient global treatment satisfaction } \\
\quad(\text { TSQM), } n\end{array}$ & 35 & 73 & 90 & 34 & 166 \\
\hline Baseline (SD) & $58.8(22.1)$ & $55.5(20.8)$ & $60.2(22.8)$ & $54.2(21.7)$ & $54.1(19.5)$ \\
\hline Endpoint (SD) & $57.6(25.2)$ & $68.6(24.4)$ & $64.9(22.5)$ & $63.7(24.2)$ & $67.1(24.4)$ \\
\hline $\begin{array}{l}\text { Mean change from baseline } \\
\text { to endpoint (SD) }\end{array}$ & $-1.2(32.2)$ & $13.1(26.0)$ & $4.8(24.7)$ & $9.5(28.9)$ & $13.1(30.0)$ \\
\hline $\begin{array}{l}95 \% \text { CI of mean change } \\
\left(p \text { value }^{\mathrm{b}}\right)\end{array}$ & $\begin{array}{l}-12.3,9.8 \\
(0.7593)\end{array}$ & $\begin{array}{l}7.1,19.2 \\
(<0.0001)\end{array}$ & $\begin{array}{r}-0.4,9.9 \\
(0.0571)\end{array}$ & $\begin{array}{l}-0.6,19.5 \\
(0.0465)\end{array}$ & $\begin{array}{l}8.5,17.7 \\
(<0.0001)\end{array}$ \\
\hline
\end{tabular}

Only patients with a valid baseline measurement and at least one valid follow-up assessment were included

ARI aripiprazole; CGI-S Clinical Global Impression-Severity; $C I$ confidence interval; ITT intent to treat; Mini-ICF-APP Mini International Classification of Functionality, Disability and Health Rating for Activity and Participation Disorders in Psychological Illnesses; OLA olanzapine; Pali ER paliperidone extended-release; PANSS Positive and Negative Syndrome Scale; PP1M once-monthly paliperidone palmitate; PSP Personal and Social Performance; QUE quetiapine; RIS risperidone; SD standard deviation; TSQM Treatment Satisfaction Questionnaire for Medication

${ }^{a}$ For the CGI-S a lower score indicates improvement; For the PSP, a higher score indicates improvement

${ }^{\mathrm{b}}$ Within-group difference was tested using the Wilcoxon signed-rank test

${ }^{\mathrm{c}}$ For the Mini-ICF-APP, a lower score indicates improvement

Investigators reported hyperprolactinemia in two patients in the OLA group and three patients in the prior Pali ER group and an increase in blood prolactin in two patients in the prior ARI group.
Of 1058 PALMFlexS patients, 177 did not have source documentation on PP1M/RIS exposure. A total 172 patients tolerated the oral tolerance test well, two patients did not, and in three patients, the test result was not available. 
Table 3 Treatment-emergent adverse events (safety ITT population; $N=472$ )

\begin{tabular}{|c|c|c|c|c|c|}
\hline Patients switched to PP1M from & $\operatorname{ARI}(n=46)$ & OLA $(n=87)$ & Pali ER $(n=104)$ & QUE $(n=44)$ & $\operatorname{RIS}(n=191)$ \\
\hline Total number of TEAEs ${ }^{\mathrm{a}}$ & 135 & 178 & 158 & 104 & 241 \\
\hline Mild (\%) & $74(54.8)$ & $112(62.9)$ & $106(67.1)$ & $53(51.0)$ & $134(55.6)$ \\
\hline Moderate $(\%)$ & $52(38.5)$ & $56(31.5)$ & $43(27.2)$ & $46(44.2)$ & $87(36.1)$ \\
\hline Severe $(\%)$ & $9(6.7)$ & $10(5.6)$ & $9(5.7)$ & $5(4.8)$ & $20(8.3)$ \\
\hline Subjects with $\geq 1 \mathrm{TEAE}^{\mathrm{a}}, n(\%)$ & $24(52.2)$ & $45(51.7)$ & $39(37.5)$ & $19(43.2)$ & $53(27.7)$ \\
\hline \multicolumn{6}{|c|}{ TEAEs ${ }^{\mathrm{a}}$ occurring in $\geq 5 \%$ of patients in any group, $n(\%)$} \\
\hline Injection-site pain & $3(6.5)$ & $12(13.8)$ & $15(14.4)$ & $7(15.9)$ & $16(8.4)$ \\
\hline Akathisia & $4(8.7)$ & $5(5.7)$ & $5(4.8)$ & $2(4.5)$ & $5(2.6)$ \\
\hline Somnolence & $2(4.3)$ & $5(5.7)$ & $2(1.9)$ & $4(9.1)$ & $4(2.1)$ \\
\hline Abnormal weight gain ${ }^{\mathrm{b}}$ & $3(6.5)$ & $1(1.1)$ & $0(0.0)$ & $2(4.5)$ & $3(1.6)$ \\
\hline Weight increased $^{\mathrm{b}}$ & $3(6.5)$ & $1(1.1)$ & $3(2.9)$ & $4(9.1)$ & $3(1.6)$ \\
\hline Insomnia & $0(0.0)$ & $7(8.0)$ & $1(1.0)$ & $2(4.5)$ & $2(1.0)$ \\
\hline Psychotic disorders & $1(2.2)$ & $6(6.9)$ & $4(3.8)$ & $1(2.3)$ & $1(0.5)$ \\
\hline
\end{tabular}

$A R I$ aripiprazole, ITT intent to treat, MedDRA Medical Dictionary for Regulatory Activities, OLA olanzapine, Pali ER paliperidone extended-release, $P P 1 M$ once-monthly paliperidone palmitate, QUE quetiapine, RIS risperidone, TEAE treatment-emergent adverse event

${ }^{\text {a }}$ Possibly, probably, or very likely related to PP1M treatment

${ }^{\mathrm{b}}$ Based on the MedDRA coding system, both terms were applicable for coding of TEAEs. None of the identified subjects were recorded under both terms simultaneously

\section{Discussion}

In this analysis, patients with non-acute schizophrenia who were switched from oral atypical antipsychotic monotherapy to PP1M demonstrated significant and clinically relevant improvements in psychotic symptoms and functioning, regardless of the previous oral atypical antipsychotic monotherapy.

At baseline, patients were mildly to moderately symptomatic, and as such, they could be considered stable but sub-optimally

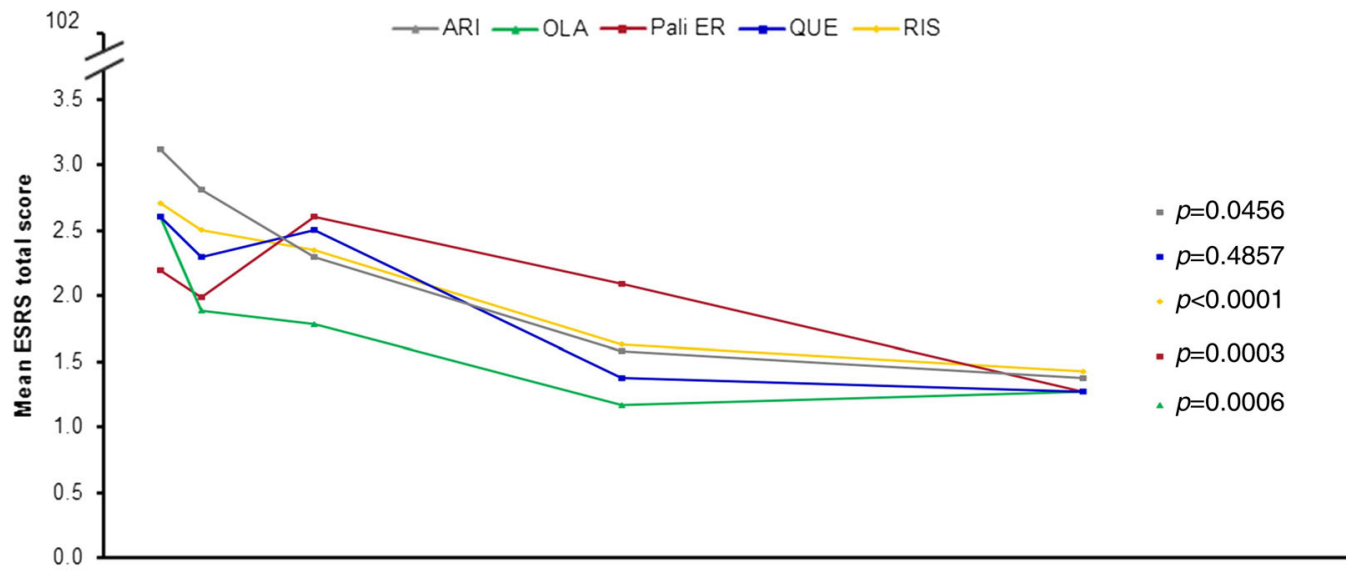

\begin{tabular}{|c|c|c|c|c|c|c|}
\hline $\begin{array}{l}\text { Patients switched } \\
\text { to PP1M from: }\end{array}$ & $\begin{array}{l}\text { Day } 1 \\
\text { (BL) }\end{array}$ & $\begin{array}{c}\text { Day } \\
8\end{array}$ & $\begin{array}{c}\text { Month } \\
1\end{array}$ & $\begin{array}{l}\text { Month } \\
3\end{array}$ & $\begin{array}{l}\text { Month } \\
\quad 6\end{array}$ & Endpoint \\
\hline $\mathrm{ARI}, \mathrm{n}$ & 46 & 44 & 41 & 37 & 31 & 46 \\
\hline OLA, n & 87 & 87 & 80 & 70 & 54 & 87 \\
\hline Pali ER, n & 104 & 102 & 100 & 93 & 82 & 104 \\
\hline QUE, $n$ & 43 & 43 & 42 & 36 & 31 & 43 \\
\hline RIS, $n$ & 186 & 183 & 183 & 162 & 151 & 186 \\
\hline
\end{tabular}

Fig. 3 Mean ESRS total score over time (safety ITT population; $N=472$ ). $A R I$ aripiprazole, $B L$ baseline, ESRS Extrapyramidal Symptom Rating Scale, ITT intent to treat, OLA olanzapine, PPIM once-monthly paliperidone palmitate, Pali ER paliperidone extendedrelease, $Q U E$ quetiapine, RIS risperidone $p$ values represent are for change from baseline to endpoint, Wilcoxon-signed-rank test 
controlled. After 6 months of treatment with PP1M, over half of all patients showed a treatment response, defined a priori as a reduction in total PANSS score of $\geq 20 \%$, which represents a clinically meaningful improvement (Cook et al. 2002) for nonacute patients. Improved efficacy of $\geq 20 \%$ was specified as the primary endpoint in this subgroup of patients, as they were considered previously stable by their treating physician for at least 1 month prior to enrolment while being prescribed an adequate dose of an oral atypical antipsychotic monotherapy. Therefore, the improvement would not be expected to be comparable with what generally would be observed in acutely ill patients, where $\geq 30$ or $\geq 50 \%$ improvements in PANSS total score are considered more adequate (Leucht 2014; Leucht et al. 2005). Nevertheless, a $\geq 50 \%$ reduction in PANSS total score was achieved by approximately one quarter of patients. The present results are consistent with a recent naturalistic study where patients previously unsuccessfully treated with oral antipsychotic medications were switched to an atypical LAT (RIS) and showed significant improvements in hospitalization days and psychotic symptoms (Schreiner et al. 2014b). However, between 17 and $32 \%$ of patients who were unsuccessfully treated with other LAI antipsychotics achieved a $\geq 50 \%$ improvement in their PANSS total score after switching to PP1M (Schreiner et al. 2015b).

PP1M maintenance doses were relatively homogeneously distributed across the groups independent of the previous oral antipsychotic and in line with those expected based on the prior mean oral doses. Exceptions were the higher maintenance doses following a switch from QUE, possibly to compensate for the loss of sedating effects and the lower maintenance doses in patients with prior ARI use, who represented the youngest patients and those with the shortest time since diagnosis, who were therefore likely to require relatively lower antipsychotic doses.

Differential outcomes were observed in efficacy, functioning, EPMS improvement, and weight gain, depending on the previous oral antipsychotic monotherapy received prior to switching to PP1M and consistent with the heterogeneity existing within the group of atypical antipsychotics. When patients were assessed according to previous oral antipsychotic monotherapy, improvements in efficacy and functioning scores were numerically greater for those patients who switched to PP1M from RIS than from other oral antipsychotics. With regard to EPMS, patients from all groups other than QUE experienced significant improvements, with the greatest improvements observed for those patients switching from oral RIS and OLA. Patients switching to PP1M from previous oral monotherapy with QUE and ARI showed greater weight gain compared with other oral antipsychotic switch groups. However, caution should be exercised when interpreting these differences as these were exploratory analyses only and the choice of the previous oral antipsychotic medication actually may have been influenced by the propensity of a patient to develop weight gain or
EPMS, which would be supported by the observation that patients previously treated with oral ARI had the highest baseline body weight and BMI.

The current analysis supports the results from previous fixed-dose RCTs and confirms the efficacy of PP1M in the treatment of schizophrenia (Gopal et al. 2010; Pandina et al. 2010), and further expands on results from retrospective and prospective studies in stabilized patients with schizophrenia that demonstrated improvements in clinical symptoms and functioning when switched from an oral to a long-acting antipsychotic medication (Möller et al. 2005; Olivares et al. 2009a; Rosa et al. 2012), as well as from a LAI antipsychotic to PP1M (Schreiner et al. 2015b).

Poor adherence to antipsychotic medication impacts the management of schizophrenia; therefore, strategies aimed at improving treatment adherence are important to achieve optimal long-term clinical outcomes (Cañas et al. 2013). LAI antipsychotics offer a number of potential benefits in long-term maintenance treatment of patients with schizophrenia, providing assured delivery of medication, regular contact with the healthcare team, and transparency about the treatment schedule, as healthcare professionals will immediately be aware if a patient misses a dose appointment (Cañas et al. 2013). A recent Italian survey reported that psychiatrists consider that switching patients to a LAI antipsychotic is a suitable approach to improve adherence in patients who are not optimally controlled with an oral atypical antipsychotic (de Bartolomeis et al. 2016). In this context, the findings from the current analysis provide evidence of the potential impact of switching to LAT with paliperidone palmitate in a real-world setting with a heterogeneous patient population representative of clinical practice.

Naturalistic studies such as this one reflect everyday clinical settings more accurately than RCTs but are subject to a number of limitations that should be kept in mind when interpreting the findings. The study was not designed to detect differential effects of previous oral antipsychotic monotherapy on patient outcomes, and therefore, the post hoc data presented herein with respect to such data should be considered exploratory in nature. Instead, a prospective pragmatic study design would enable analyses of the effectiveness and safety of PP1M when switching from different oral antipsychotic monotherapies. The study was open-label with no active comparator group, and as such, these data do not provide a headto-head comparison between treatments; rather, they suggest that failure with one antipsychotic medication does not predict failure with another. Increased time spent in the study and with healthcare professionals may have added to the observed improvement in outcomes.

Other limitations associated with an open-label design also impact the interpretation and generalizability of the study results. As neither the patients nor their physicians were blinded to the change in antipsychotic treatment, potential bias may have been introduced, affecting both patient- and physician- 
reported subjective outcome measures (such as PANSS and PSP scores, and both patient and physician treatment satisfaction scores).

Although this particular study did not have an oral control group, a recent relapse prevention study showed that in patients recently diagnosed with schizophrenia, treatment with PP1M almost doubled the time to relapse compared with treatment with oral antipsychotics (Schreiner et al. 2015a). The present study provides important information about the potential expectations for treatment outcomes in a clinically representative population (many of whom would not have been eligible for inclusion in RCTs) after switching from atypical oral antipsychotic monotherapy to PP1M in a more realworld environment, and includes clinically relevant insights into dosage selection and adjustments and the use of concomitant medication.

\section{Conclusions}

In conclusion, these data illustrate that non-acute patients (considered clinically stable by their physician) with schizophrenia may show meaningful improvement of psychotic symptoms, functioning, and treatment satisfaction when switched from oral atypical antipsychotic monotherapy to long-acting treatment with PP1M. However, this open-label, naturalistic study did not have an oral control group, limiting the strength of these findings. Validation of these observations in a blinded study with suitable comparator group(s) would be a valuable next step. In this diverse patient population, PP1M was well tolerated, presenting no new safety signals.

Acknowledgments Medical writing support was provided by ApotheCom, London, UK and funded by Janssen Cilag. This study was funded by Janssen Cilag.

\section{Compliance with ethical standards}

Conflict of interest A Schreiner, P Bergmans, P Cherubin, S Keim, and L Hargarter are employees of Janssen Cilag. A Caspi, S Suleman, and E Lara have no conflicts of interest to declare. I Pinchuk has received speaker's honoraria from Pfizer. D Schuepbach has acted as a consultant for Janssen Cilag, Lundbeck, and Takeda; has received speaker's honoraria from Lundbeck and Takeda; and was a principal investigator for a Janssen Cilag-funded study.

Open Access This article is distributed under the terms of the Creative Commons Attribution 4.0 International License (http:// creativecommons.org/licenses/by/4.0/), which permits unrestricted use, distribution, and reproduction in any medium, provided you give appropriate credit to the original author(s) and the source, provide a link to the Creative Commons license, and indicate if changes were made.

\section{References}

Alphs L, Schooler N, Lauriello J (2014) How study designs influence comparative effectiveness outcomes: the case of oral versus longacting injectable antipsychotic treatments for schizophrenia. Schizophr Res 156:228-232

Atkinson MJ, Sinha A, Hass SL et al (2004) Validation of a general measure of treatment satisfaction, the Treatment Satisfaction Questionnaire for Medication (TSQM), using a national panel study of chronic disease. Health Qual Life Outcomes 2:12

Barnes TR (2011) Evidence-based guidelines for the pharmacological treatment of schizophrenia: recommendations from the British Association for Psychopharmacology. J Psychopharmacol (Oxford, England) 25: 567-620

Cañas F, Alptekin K, Azorin JM et al (2013) Improving treatment adherence in your patients with schizophrenia: the STAY initiative. Clin Drug Investig 33:97-107

Carey KB, Cocco KM, Simons JS (1996) Concurrent validity of clinicians' ratings of substance abuse among psychiatric outpatients. Psychiatr Serv (Washington, DC) 47: 842-7

Chouinard G, Margolese HC (2005) Manual for the Extrapyramidal Symptom Rating Scale (ESRS). Schizophr Res 76:247-265

Cook PE, Goldberg JO, Van Lieshout RJ (2002) Benefits of switching from typical to atypical antipsychotic medications: a longitudinal study in a community-based setting. Can J Psychiatr 47:870-874

Davis JM, Chen N, Glick ID (2003) A meta-analysis of the efficacy of second-generation antipsychotics. Arch Gen Psychiatry 60:553-564

de Bartolomeis A, Fagiolini A, Vaggi M et al (2016) Targets, attitudes, and goals of psychiatrists treating patients with schizophrenia: key outcome drivers, role of quality of life, and place of long-acting antipsychotics. Neuropsychiatr Dis Treat 12:99-108

De Hert M, Yu W, Detraux J et al (2012) Body weight and metabolic adverse effects of asenapine, iloperidone, lurasidone and paliperidone in the treatment of schizophrenia and bipolar disorder: a systematic review and exploratory meta-analysis. CNS drugs 26:733-759

Gopal S, Hough DW, Xu H et al (2010) Efficacy and safety of paliperidone palmitate in adult patients with acutely symptomatic schizophrenia: a randomized, double-blind, placebo-controlled, dose-response study. Int Clin Psychopharmacol 25:247-256

Hargarter L, Cherubin P, Bergmans P et al (2015) Intramuscular longacting paliperidone palmitate in acute patients with schizophrenia unsuccessfully treated with oral antipsychotics. Prog NeuroPsychopharmacol Biol Psychiatry 58:1-7

Hasan A, Falkai P, Wobrock T et al (2013) World Federation of Societies of Biological Psychiatry (WFSBP) guidelines for biological treatment of schizophrenia, part 2: update 2012 on the long-term treatment of schizophrenia and management of antipsychotic-induced side effects. World J Biol Psychiatry 14:2-44

Higashi K, Medic G, Littlewood KJ et al (2013) Medication adherence in schizophrenia: factors influencing adherence and consequences of nonadherence, a systematic literature review. Ther Adv Psychopharmacol 3:200-218

Janssen Cilag (2015) Paliperidone palmitate summary of product characteristics. Available at: http://www.ema.europa.eu/docs/en GB/document_library/EPAR_-_Product_Information/human/00210 $\overline{5}$ /WC500103317.pdf (Accessed October 2016)

Jones MP, Nicholl D, Trakas K (2010) Efficacy and tolerability of paliperidone ER and other oral atypical antipsychotics in schizophrenia. Int J Clin Pharmacol Ther 48:383-399

Kahn RS, Fleischhacker WW, Boter H et al (2008) Effectiveness of antipsychotic drugs in first-episode schizophrenia and schizophreniform disorder: an open randomised clinical trial. Lancet 371:1085-1097

Kirson NY, Weiden PJ, Yermakov S et al (2013) Efficacy and effectiveness of depot versus oral antipsychotics in schizophrenia: 
synthesizing results across different research designs. J Clin Psychiatry 74: 568-75

Kishimoto T, Nitta M, Borenstein M et al (2013) Long-acting injectable versus oral antipsychotics in schizophrenia: a systematic review and meta-analysis of mirror-image studies. J Clin Psychiatry 74: 957-65

Kishimoto T, Robenzadeh A, Leucht C et al (2014) Long-acting injectable vs oral antipsychotics for relapse prevention in schizophrenia: a meta-analysis of randomized trials. Schizophr Bull 40:192-213

Kozma CM, Weiden PJ (2009) Partial compliance with antipsychotics increases mental health hospitalizations in schizophrenic patients: analysis of a national managed care database. Am Health Drug Benefits 2:31-38

Lerma-Carrillo I, de Pablo Bruhlmann S, del Pozo ML et al (2008) Antipsychotic polypharmacy in patients with schizophrenia in a brief hospitalization unit. Clin Neuropharmacol 31:319-332

Leucht S (2014) Measurements of response, remission, and recovery in schizophrenia and examples for their clinical application. J Clin Psychiatry 75 Suppl 1: 8-14

Leucht S, Heres S (2006) Epidemiology, clinical consequences, and psychosocial treatment of nonadherence in schizophrenia. J Clin Psychiatry 67 Suppl 5: 3-8

Leucht S, Kane JM, Kissling W et al (2005) What does the PANSS mean? Schizophr Res 79:231-238

Leucht C, Heres S, Kane JM et al (2011) Oral versus depot antipsychotic drugs for schizophrenia - a critical systematic review and metaanalysis of randomised long-term trials. Schizophr Res 127:83-92

Leucht S, Cipriani A, Spineli L et al (2013) Comparative efficacy and tolerability of 15 antipsychotic drugs in schizophrenia: a multipletreatments meta-analysis. Lancet 382:951-962

Lieberman JA, Stroup TS, McEvoy JP et al (2005) Effectiveness of antipsychotic drugs in patients with chronic schizophrenia. N Engl J Med 353:1209-1223

Linden M, Baron S (2005) The "Mini-ICF-Rating for Mental Disorders (Mini-ICF-P)". A short instrument for the assessment of disabilities in mental disorders. Die Rehabilitation 44:144-151

Liu-Seifert H, Adams DH, Kinon BJ (2005) Discontinuation of treatment of schizophrenic patients is driven by poor symptom response: a pooled post-hoc analysis of four atypical antipsychotic drugs. BMC Med 3:21

Llorca P-M (2008) Partial compliance in schizophrenia and the impact on patient outcomes. Psychiatry Res 161:235-247

Möller H-J, Llorca P-M, Sacchetti E et al (2005) Efficacy and safety of direct transition to risperidone long-acting injectable in patients treated with various antipsychotic therapies. Int $\mathrm{Clin}$ Psychopharmacol 20:121-130

Molodynski A, Linden M, Juckel G et al (2013) The reliability, validity, and applicability of an English language version of the Mini-ICFAPP. Soc Psychiatry Psychiatr Epidemiol 48:1347-1354

Morosini PL, Magliano L, Brambilla L et al (2000) Development, reliability and acceptability of a new version of the DSM-IV Social and Occupational Functioning Assessment Scale (SOFAS) to assess routine social functioning. Acta Psychiatr Scand 101: 323-9

Naber D, Lambert M (2009) The CATIE and CUtLASS studies in schizophrenia: results and implications for clinicians. CNS drugs 23:649659
National Institute for Health and Care Excellence (NICE) (2014) Psychosis and schizophrenia in adults: treatment and management (CG178)

Olivares JM, Peuskens J, Pecenak J et al (2009a) Clinical and resourceuse outcomes of risperidone long-acting injection in recent and longterm diagnosed schizophrenia patients: results from a multinational electronic registry. Curr Med Res Opin 25:2197-2206

Olivares JM, Rodriguez-Morales A, Diels J et al (2009b) Long-term outcomes in patients with schizophrenia treated with risperidone long-acting injection or oral antipsychotics in Spain: results from the electronic Schizophrenia Treatment Adherence Registry (eSTAR). Eur Psychiatry 24:287-296

Pandina GJ, Lindenmayer J-P, Lull J et al (2010) A randomized, placebocontrolled study to assess the efficacy and safety of 3 doses of paliperidone palmitate in adults with acutely exacerbated schizophrenia. J Clin Psychopharmacol 30:235-244

Robinson D, Woerner MG, Alvir JM et al (1999) Predictors of relapse following response from a first episode of schizophrenia or schizoaffective disorder. Arch Gen Psychiatry 56:241-247

Rosa F, Schreiner A, Thomas P et al (2012) Switching patients with stable schizophrenia or schizoaffective disorder from olanzapine to risperidone long-acting injectable. Clin Drug Investig 32:267-279

Rummel-Kluge C, Komossa K, Schwarz S et al (2010) Head-to-head comparisons of metabolic side effects of second generation antipsychotics in the treatment of schizophrenia: a systematic review and meta-analysis. Schizophr Res 123:225-233

Schreiner A, Bergmans P, Cherubin P et al (2014a) A prospective flexible-dose study of paliperidone palmitate in nonacute but symptomatic patients with schizophrenia previously unsuccessfully treated with oral antipsychotic agents. Clin Ther 36:1372-88.e1

Schreiner A, Svensson A, Wapenaar R et al (2014b) Long-acting injectable risperidone and oral antipsychotics in patients with schizophrenia: results from a prospective, 1-year, non-interventional study World J Biol Psychiatry 15:534-545

Schreiner A, Aadamsoo K, Altamura AC et al (2015a) Paliperidone palmitate versus oral antipsychotics in recently diagnosed schizophrenia. Schizophr Res 169:393-399

Schreiner A, Bergmans P, Cherubin P et al (2015b) Paliperidone palmitate in non-acute patients with schizophrenia previously unsuccessfully treated with risperidone long-acting therapy or frequently used conventional depot antipsychotics. J Psychopharmacol (Oxford, England) 29: 910-22

Tandon R, Marcus RN, Stock EG et al (2006) A prospective, multicenter, randomized, parallel-group, open-label study of aripiprazole in the management of patients with schizophrenia or schizoaffective disorder in general psychiatric practice: Broad Effectiveness Trial With Aripiprazole (BETA). Schizophr Res 84:77-89

Taylor D, Paton C, Kapur S (2012) The Maudsley prescribing guidelines in psychiatry, 11th edn. Wiley-Blackwell

Tiihonen J, Haukka J, Taylor M et al (2011) A nationwide cohort study of oral and depot antipsychotics after first hospitalization for schizophrenia. Am J Psychiatry 168:603-609 